\title{
Role of caveolin-3 in lymphocyte activation
}

\author{
Chinh Tran ${ }^{a,}{ }^{,}$, Creed M. Stary ${ }^{b,{ }^{*}}$, Jan M. Schilling ${ }^{a}$, Brandon Bentley ${ }^{a}$, Hemal H. Patel ${ }^{a}$, and \\ David M. Roth ${ }^{\mathrm{a}}$ \\ a Department of Anesthesiology, Veterans Affairs San Diego Healthcare System, University of \\ California, San Diego, La Jolla, California \\ b Department of Anesthesia, Stanford University, Stanford, California
}

\section{Abstract}

\begin{abstract}
Aims-Caveolins are structural proteins clustered in lipid-rich regions of plasma membrane involved in coordinating signal transduction in various organ systems. While caveolin-1 (Cav-1) has been shown to regulate lymphocyte activation, the role of caveolin-3 (Cav-3) in immune system signaling has not been investigated. We tested the hypothesis that Cav-3 modulates lymphocyte activation.
\end{abstract}

Main methods-Lymphocyte/leukocyte subpopulations from WT and Cav-3 mice were profiled with flow cytometry. Cytokine production in quiescent and activated splenocytes from WT and Cav-3 mice was assessed with ELISA.

Key findings-Levels of T-cells, monocytes, and natural killer cells were not different between WT and KO mice, however KO mice had lower B-cell population-percentage. Functionally, activated lymphocytes from Cav-3 KO mice demonstrated significantly reduced expression of IL-2 compared to WT, while expression of TNFa, IL-6, and IL-10 was not different. Finally, expression of IL-17 was significantly reduced in T-helper cells from KO mice, while IFN $\gamma$ was not, suggesting that Cav-3 is a determinant in the development of the Th-17 subpopulation.

Significance-This study is the first to demonstrate that Cav-3 may be a novel participant in Bcell expression, T-cell cytokine production and activation of inflammation.

\section{Keywords}

cytokine; inflammation; leukocyte; immune system; signaling

\section{Introduction}

Membrane lipid rafts (MLRs) are discreet microdomains of the cell membrane that concentrate and localize cellular signaling molecules by providing a lipid-rich (i.e.,

\footnotetext{
Address Correspondence to: David M. Roth, MD, PhD Veterans Affairs Healthcare System Medical Center (125) 3350 La Jolla Village Drive San Diego, CA 92161-5085 droth@ucsd.edu.

* Considered primary authors

Conflict of Interest Statement

The authors declare that they have no conflicts of interest.

The authors have no additional financial disclosures.
} 
sphingomyelin, glycosphingolipids, and cholesterol) platform for protein anchoring. By providing a stable binding environment for protein-protein interactions, MLRs promote a variety of physiological functions such as cell surface signaling (Lisanti et al., 1994, Ostrom et al., 2001, Steinberg and Brunton, 2001), endocytosis (Anderson, 1993), calcium homeostasis (Fujimoto et al., 1992, Fujimoto, 1993, Scriven et al., 2002) and intracellular cholesterol transport (Murata et al., 1995). Caveolae, morphologic invaginations of the cell membrane, are a subset of MLRs rich in the structural/scaffolding proteins caveolins (Smart et al., 1996), a family of proteins approximately $17-24 \mathrm{kDa}$ in size that exist in three isoforms (Cav-1, -2 , and -3). Cav-1 is essential for formation of caveolae in endothelial cells, fibroblasts, and pneumocytes (Smart et al., 1999), whereas Cav-2 plays an unclear but likely supportive role by forming hetero-oligomers with Cav-1 (Smart et al., 1999, Razani et al., 2001). Cav-3 KO mice are viable but subject to skeletal and cardiac myopathies (Monier et al., 1995, Hagiwara et al., 2000), and our laboratory and others have demonstrated a critical role for caveolin in protection from ischemia-reperfusion injury (Bromley et al., 2001, Galbiati et al., 2001, Chidlow and Sessa, 2010, Stary et al., 2012). MLR-mediated signal transduction is an important element in the activation of the immune system (Gargalovic and Dory, 2003, Ohnuma et al., 2004, 2009, Sawada et al., 2010, Fu et al., 2012) and inflammatory response (Oakley et al., 2009, Garrean et al., 2006, Feng et al., 2010, Xu et al., 2010, Hu et al., 2008), however this previous work has largely focused on the more ubiquitously expressed Cav-1 isoform. In contrast, the role of Cav-3 in immune system signaling has been relatively under-investigated. Therefore, in the present study we utilized global Cav-3 KO mice to define the role of Cav-3 in immune system signaling by testing the hypothesis that Cav-3 participates in physiologic T-cell activation.

\section{Materials and Methods}

\section{Animals}

Animals (total $\mathrm{n}=35$ ) were treated in compliance with the Guide for the Care and Use of Laboratory Animals (National Academy of Science). All protocols were approved by the Veterans Affairs San Diego Healthcare System Institutional Animal Care and Use Committee. Animals were kept on a 12-h light/dark cycle in a temperature-controlled room with ad libitum access to food and water. The genotype of Cav-3 KO mice was confirmed by PCR.

Lymphocyte Isolation-Eight- to ten-week-old Cav-3 KO (13) mice $(\mathrm{n}=19)$ or agematched C57BL/6 wild type (WT) controls $(\mathrm{n}=16)$ were euthanized and spleens harvested and macerated through a $70 \mu \mathrm{m}$ cell strainer (Fisher Scientific). Residual red blood cells were lysed with $5 \mathrm{~mL}$ ammonium-chloride-potassium (ACK) lysis buffer (Life Technologies) for 5 minutes at room temperature. The lymphocytes were then washed and pelleted twice before being resuspended in RPMI media (Invitrogen) supplemented with $10 \%$ fetal calf serum (FCS, Gibco), $2 \mathrm{mM}$ glutamine (Sigma-Aldrich), $50 \mathrm{U} / \mathrm{mL}$ penicillin (Sigma-Aldrich), $50 \mu \mathrm{g} / \mathrm{mL}$ streptomycin (Sigma-Aldrich), $0.6 \mathrm{mM}$ sodium pyruvate (Sigma-Aldrich), $1 \mathrm{mM}$ HEPES (Sigma-Aldrich), and $0.055 \mathrm{mM} \beta$-mercapthoethanol (Sigma-Aldrich). 
Flow Cytometry-Rat anti-mouse CD3 FITC antibody (561798, BD Pharmigen), CD14 FITC (11-0141-82, eBioscience), CD16 FITC (11-0161-82, eBiosciences), CD19 FITC (11-0193-82, eBioscience), and Rat IgG FITC control (556923, BD Pharmigen) were used to stain splenocytes for flow cytometric analysis. $3 \times 10^{6}$ splenocytes were pelleted and resuspended in the 1:100 dilution of antibodies and 2\% FCS:PBS (Gibco), then incubated for $30 \mathrm{~min}$ on ice. The stained cells were washed and pelleted 3 times with $2 \%$ FCS: PBS. Finally cells were fixed in 1\% paraformaldehyde:PBS (Fisher Scientific) before flow analysis in a Becton Dickinson FACSCalibur flow cytometer (Flow Cytometry Research Core at the VA San Diego).

T-cell Activation-12 well plates were coated with $5 \mu \mathrm{g} / \mathrm{mL}$ rat anti-mouse CD3 antibody (16-0032-85, eBioscience) and $5 \mu \mathrm{g} / \mathrm{mL}$ CD28 (16-0281-85, eBioscience) in PBS (Invitrogen) overnight at $4^{\circ} \mathrm{C} .10 \mu \mathrm{g} / \mathrm{mL}$ rat Ig (16-4301-85, eBiopscience) in PBS was used as a control. After antibody coating, the plates were washed once with PBS. $4 \times 10^{6}$ freshly isolated lymphocytes were then plated into wells with RPMI media (Gibco). Lymphocytes were then cultured at $36^{\circ} \mathrm{C}$ for $48 \mathrm{hrs}$.

Cytokine Detection-Supernantants were isolated after T-cells were stimulated by antibody and diluted 1:100 or 1:1000 final concentration for assay by enzyme-linked immunosorbent assay (ELISA). ELISAs (Life Biosciences) for IL-2, IL-17, IL-6, IL-10, INF $\gamma$, and TNFa were utilized according to the manufacturer's protocols. Data was acquired via Tecan Plate Reader Infinite M200.

Statistics-All data were analyzed via Mann Whitney U test. Significance was set at $\mathrm{p}<$ 0.05 . All data are presented as mean \pm SEM. All statistical analysis was performed using Prism 6 (GraphPad Software, Inc).

\section{Results}

\section{Lymphocyte Populations}

Lymphocyte/leukocyte subpopulations of T-cells, B-cells, monocytes and natural killer cells were determined in WT and Cav-3 KO mice using flow cytometry (Fig. 1). We observed a decrease in the population distribution of B-cells in Cav-3 KO mice relative to WT mice; however, T-cell, monocyte, and natural killer cell populations were not altered.

\section{T-cell Activation and Cytokine Production}

We sought to evaluate the cytokine production from splenic lymphocytes harvested from WT and Cav-3 KO mice after T-cell activation (Fig. 2) using stimulating anti-CD3 and antiCD28 antibodies. Cytokine levels from supernatants of cells treated with stimulating antibodies were compared with those of nonspecific control antibody. For each experimental set, the cytokine levels were normalized to the average WT level to reduce variation between experiments. We found that IL-2 and IL-17 production were significantly reduced in Cav-3 KO mice relative to WT. No differences in IFN $\gamma$, TNFa, IL-6, and IL-10 levels were observed. 


\section{Discussion}

The potential role for Cav-3 protein in the immune response has never been investigated. In our experiments we observed that levels of T-cells, monocytes, and natural killer cells were not different between WT and KO mice, however KO mice had significantly fewer B-cells. Additionally, we observed that production of the activating cytokine IL-2 after T-cell stimulation with anti-CD3 and anti-CD28 antibodies was reduced. This suggests that Cav-3 signaling is at least partially involved in the production of this cytokine. However, we did not find that production of the Th-1 specific cytokine (IFN $\gamma$ ), the proinflammatory cytokines (TNFa, IL-6), nor the inhibitory cytokine (IL-10) were significantly affected by the lack of Cav-3 expression. Taken together this suggests that Cav-3 may be important for initial Tcell activation by elaboration of IL-2, and that either lowered IL-2 levels and/or Cav-3 itself, may also be important for subsequent production of the inflammatory mediator IL-17. How this relates to the overall pro- or anti-inflammatory state remains to be elucidated, as other cytokine levels we measured appeared to be unaffected by the lack of Cav-3 expression, even in the face of reduced baseline IL-2 production.

Previous work regarding a role for MLR and caveolin mediated signal transduction in activation of the immune system and the inflammatory response has focused on the Cav-1 isoform, demonstrating a role in T-cell activation by upregulation of CD86 on antigen presenting cells (Ohnuma et al., 2004), and the T-cell recall-response to antigen (Ohnuma et al., 2009). Tomassian et al. (Tomassian et al., 2011) demonstrated that Cav-1 is essential to coordinate an appropriate T-cell response to antigen, while Fu et. al (2012) observed that Cav-1 is necessary for the transformation of quiescent monocytes to active macrophages. Upregulation of Cav-1 has been observed in adult T-cell leukemia cells (Sawada et al., 2010) and macrophages undergoing apoptosis (Gargalovic and Dory, 2003), and Cav-1 has been shown to play a critical role in induction of inflammatory pathways via interleukin- $1 \beta$ (IL-1 $\beta$ ) signaling and NFkB activation (Garrean et al., 2006). In the respiratory system, alveolar macrophages with an inflammatory phenotype were associated with enhanced expression of Cav-1 (Xu et al., 2010). Cav-1 knockout mice (KO) showed reduced LPS driven lung inflammation (Garrean et al., 2006) and reduced activation, adhesion, and transendothelial migration of neutrophils (Hu et al., 2008). Finally, Cav-1 KO mice demonstrated reduced survival in an ischemic-bowel model of sepsis, associated with an enhanced expression of TNFa and IL-6 (Feng et al., 2010).

Although Cav-1 appears to play a prominent role in immune system signaling, there is limited information regarding a role for Cav-3. Cav-3 has been detected in bovine lymphocytes (Harris et al., 2002), but to date the specific role of Cav-3 in lymphocyte activation had not been investigated. Cav-3 expression was thought to be restricted to skeletal, cardiac, and some smooth muscle (Tang et al., 1996). However we have recently demonstrated that Cav-3 is necessary in coordinating microglial activation in the brain (Niesman et al., 2013), and that Cav-3 KO mice have an enhanced neuroinflammatory response to traumatic brain injury (Niesman et al., 2014), suggesting a regulatory role for Cav-3 in immunomodulation in non-muscle cell types. Our current results suggest Cav-3 may be involved in lymphocyte differentiation, proliferation and/or apoptosis. We observed that the B-cell population appears to be reduced in Cav-3 KO mice. This could either be due 
to a disorder in B-cell development and/or an increase in B-cell apoptosis in mice lacking Cav-3. Although we have previously demonstrated that Cav-3 plays an anti-apoptotic role in cardiac myocytes (Tsutsumi et al., 2008) via induction of pro-survival Akt signaling, given the complexities of immune cell differentiation, activation and cytokine signaling heterogeneity between multiple cell types, the mechanism for the observed decrease in Bcells is likely multifactorial, and at present, it is unclear whether the reduction in the B-cell population was due to decreased differentiation/proliferation or increased apoptosis. Similarly, the reduction in IL-17 could be due to a defect in differentiation in the Th-17 subpopulation and/or and increase in apoptosis of Th-17 lymphocytes. Interestingly, levels of both IL-2 (Mingari et al., 1984) and IL-17 (Tarlinton, 2008) correlate with B-cell proliferation. T-cell differentiation and cytokine activation are both considered to be largely regulated by signal transducer and activator of transcription (STAT) proteins (Li et al., 2014). A recent study by Ryter et al. (2014) demonstrated that Cav-1 deficient mice have altered STAT-1 and STAT-3 signaling in lung fibroblasts, providing a potential mechanism of caveolin-mediated regulation of T-cell differentiation. However, whether Cav-3 plays a role in regulation of STAT pathways and subsequently T-cell activation is currently unknown. Parallel investigations utilizing Cav-3 overexpressing mouse models targeted to the immune system may yield significant insight into the precise Cav-3 mediated signaling mechanisms coordinating immune activation and differentiation.

The data from the present study indicating a role for Cav-3 in the immune signaling response may hold significant clinical relevance. For example, recombinant IL-2 has been recently approved by the FDA for treating malignant melanoma and renal cell cancer. Conversely an IL-17 inhibitor is in phase II clinical trials for the treatment of advanced colorectal cancer and refractory Hodgkin's Lymphoma, while selective inhibition of B-cell proliferation may be beneficial in the treatment of a variety of non-Hodgkin's B-cell malignancies. It would also be interesting to observe Cav-3 KO mice in immune-mediated disease models (e.g. asthma, inflammatory arthritis, psoriasis, sepsis, and stroke) in order to determine whether there is a benefit or detriment from the absence of Cav-3. However, whether Cav-3 exists in human lymphocytes and if so, whether it plays a functional role in immune-mediated diseases is presently unknown. The role of Cav-3 in lymphocyte activation and differentiation and the clinical implications therein are potential areas for future work.

\section{Conclusions}

In summary, our data are the first to suggest that the loss of Cav-3 has an impact on critical aspects of immune expression and activation. We believe that a role for Cav-3 in lymphocyte activation is a novel observation and further elucidation of the precise mechanisms and consequences of Cav-3 expression in lymphocyte activation and differentiation is warranted. As the immune response is a critical determinant of the severity of injury in a vast array of disease states, these findings may hold significant clinical relevance. Delineating the precise role of Cav-3 on activation of these pathways will be critical in utilizing Cav-3 to modulate these pathways for clinical therapies, and will be an area of future investigation. 


\section{Acknowledgements}

We would like to acknowledge Michael Migita for his technical assistance. This work was supported by Veteran Affairs Merit Awards from the Department of Veterans Affairs BX000783 (to DMR) and BX001963 (to HHP), and National Institutes of Health Grants T32-GM089626 (to CMS), HL091071 (to HHP), HL107200 (to HHP and DMR), HL066941 (to HHP and DMR) and HL115933 (to HHP and DMR).

\section{References}

Lisanti MP, Scherer PE, Tang Z, Sargiacomo M. Caveolae, caveolin and caveolin-rich membrane domains: a signalling hypothesis. Trends Cell Biol. 1994; 4:231-235. [PubMed: 14731661]

Ostrom RS, Gregorian C, Drenan RM, Xiang Y, Regan JW, Insel PA. Receptor number and caveolar co-localization determine receptor coupling efficiency to adenylyl cyclase. J Biol Chem. 2001; 276:42063-42069. [PubMed: 11533056]

Steinberg SF, Brunton LL. Compartmentation of G protein-coupled signaling pathways in cardiac myocytes. Annu Rev Pharmacol Toxicol. 2001; 41:751-773. [PubMed: 11264475]

Anderson RG. Potocytosis of small molecules and ions by caveolae. Trends Cell Biol. 1993; 3:69-72. [PubMed: 14731772]

Fujimoto T, Nakade S, Miyawaki A, Mikoshiba K, Ogawa K. Localization of inositol 1,4,5trisphosphate receptor-like protein in plasmalemmal caveolae. J Cell Biol. 1992; 119:1507-1513. [PubMed: 1334960]

Fujimoto T. Calcium pump of the plasma membrane is localized in caveolae. J Cell Biol. 1993; 120:1147-1157. [PubMed: 8382206]

Scriven DR, Klimek A, Lee KL, Moore ED. The molecular architecture of calcium microdomains in rat cardiomyocytes. Ann N Y Acad Sci. 2002; 976:488-499. [PubMed: 12502603]

Murata M, Peranen J, Schreiner R, Wieland F, Kurzchalia TV, Simons K. VIP21/caveolin is a cholesterol-binding protein. Proc Natl Acad Sci U S A. 1995; 92:10339-10343. [PubMed: 7479780]

Smart EJ, Ying Y, Donzell WC, Anderson RG. A role for caveolin in transport of cholesterol from endoplasmic reticulum to plasma membrane. J Biol Chem. 1996; 271:29427-29435. [PubMed: 8910609]

Smart EJ, et al. Caveolins, liquid-ordered domains, and signal transduction. Mol Cell Biol. 1999; 19:7289-7304. [PubMed: 10523618]

Razani B, et al. Caveolin-1 null mice are viable but show evidence of hyperproliferative and vascular abnormalities. J Biol Chem. 2001; 276:38121-38138. [PubMed: 11457855]

Monier S, Parton RG, Vogel F, Behlke J, Henske A, Kurzchalia TV. VIP21-caveolin, a membrane protein constituent of the caveolar coat, oligomerizes in vivo and in vitro. Mol Biol Cell. 1995; 6:911-927. [PubMed: 7579702]

Hagiwara Y, et al. Caveolin-3 deficiency causes muscle degeneration in mice. Hum Mol Genet. 2000; 9:3047-3054. [PubMed: 11115849]

Galbiati F, et al. Caveolin-3 null mice show a loss of caveolae, changes in the microdomain distribution of the dystrophin-glycoprotein complex, and ttubule abnormalities. J Biol Chem. 2001; 276:21425-21433. [PubMed: 11259414]

Stary CM, Tsutsumi YM, Patel PM, Head BP, Patel HH, Roth DM. Caveolins: targeting pro-survival signaling in the heart and brain. Front Physiol. 2012; 3:393. [PubMed: 23060817]

Bromley SK, et al. The immunological synapse. Annu Rev Immunol. 2001; 19:375-396. [PubMed: 11244041]

Chidlow JH Jr. Sessa WC. Caveolae, caveolins, and cavins: complex control of cellular signalling and inflammation. Cardiovasc Res. 2010; 86:219-225. [PubMed: 20202978]

Ohnuma K, et al. CD26 up-regulates expression of CD86 on antigen-presenting cells by means of caveolin-1. Proc Natl Acad Sci U S A. 2004; 101:14186-14191. [PubMed: 15353589]

Ohnuma K, et al. Blockade of CD26-mediated T cell costimulation with soluble caveolin-1-Ig fusion protein induces anergy in CD4+T cells. Biochem Biophys Res Commun. 2009; 386:327-332. [PubMed: 19523449] 
Sawada S, et al. Overexpression of caveolin-1 in adult T-cell leukemia. Blood. 2010; 115:2220-2230. [PubMed: 20061557]

Gargalovic P, Dory L. Cellular apoptosis is associated with increased caveolin-1 expression in macrophages. J Lipid Res. 2003; 44:1622-1632. [PubMed: 12777465]

$\mathrm{Fu}$ Y, et al. Caveolin-1 plays a critical role in the differentiation of monocytes into macrophages. Arterioscler Thromb Vasc Biol. 2012; 32:e117-e125. [PubMed: 22772753]

Oakley FD, Smith RL, Engelhardt JF. Lipid rafts and caveolin-1 coordinate interleukin-1beta (IL-1beta)-dependent activation of NFkappaB by controlling endocytosis of Nox 2 and IL-1beta receptor 1 from the plasma membrane. J Biol Chem. 2009; 284:33255-33264. [PubMed: 19801678]

Xu Y, Krause A, Hamai H, Harvey BG, Worgall TS, Worgall S. Proinflammatory phenotype and increased caveolin-1 in alveolar macrophages with silenced CFTR mRNA. PLoS One. 2010; 5:e11004. [PubMed: 20543983]

Garrean S, et al. Caveolin-1 regulates NF-kappaB activation and lung inflammatory response to sepsis induced by lipopolysaccharide. J Immunol. 2006; 177:4853-4860. [PubMed: 16982927]

Feng H, et al. Caveolin-1 protects against sepsis by modulating inflammatory response, alleviating bacterial burden, and suppressing thymocyte apoptosis. J Biol Chem. 2010; 285:25154-25160. [PubMed: 20534584]

Hu G, Ye RD, Dinauer MC, Malik AB, Minshall RD. Neutrophil caveolin-1 expression contributes to mechanism of lung inflammation and injury. Am J Physiol Lung Cell Mol Physiol. 2008; 294:L178-L186. [PubMed: 17993589]

Tomassian T, Humphries LA, Liu SD, Silva O, Brooks DG, Miceli MC. Caveolin-1 orchestrates TCR synaptic polarity, signal specificity, and function in CD8 T cells. J Immunol. 2011; 187:29933002. [PubMed: 21849673]

Harris J, Werling D, Koss M, Monaghan P, Taylor G, Howard CJ. Expression of caveolin by bovine lymphocytes and antigen-presenting cells. Immunology. 2002; 105:190-195. [PubMed: 11872094]

Tang Z, et al. Molecular cloning of caveolin-3, a novel member of the caveolin gene family expressed predominantly in muscle. J Biol Chem. 1996; 271:2255-2261. [PubMed: 8567687]

Niesman IR, et al. Caveolin isoform switching as a molecular, structural, and metabolic regulator of microglia. Mol Cell Neurosci. 2013; 56:283-297. [PubMed: 23851187]

Niesman IR, et al. Traumatic brain injury enhances neuroinflammation and lesion volume in caveolin deficient mice. J Neuroinflammation. 2014; 11:39. [PubMed: 24593993]

Tsutsumi YM, et al. Cardiac-specific overexpression of caveolin-3 induces endogenous cardiac protection by mimicking ischemic preconditioning. Circulation. 2008; 118:1979-1988. [PubMed: 18936328]

Mingari MC, et al. Human interleukin-2 promotes proliferation of activated B cells via surface receptors similar to those of activated T cells. Nature. 1984; 312:641-643. [PubMed: 6438535]

Tarlinton D. IL-17 drives germinal center B cells? Nat Immunol. 2008; 9:124-126. [PubMed: 18204425]

Li P, Spolski R, Liao W, Leonard WJ. Complex interactions of transcription factors in mediating cytokine biology in T cells. Immunol Rev. 2014; 261:141-156. [PubMed: 25123282]

Ryter SW, Choi AM, Kim HP. Profibrogenic phenotype in caveolin-1 deficiency via differential regulation of STAT-1/3 proteins. Biochem Cell Biol. 2014; 92:370-378. [PubMed: 25263949] 

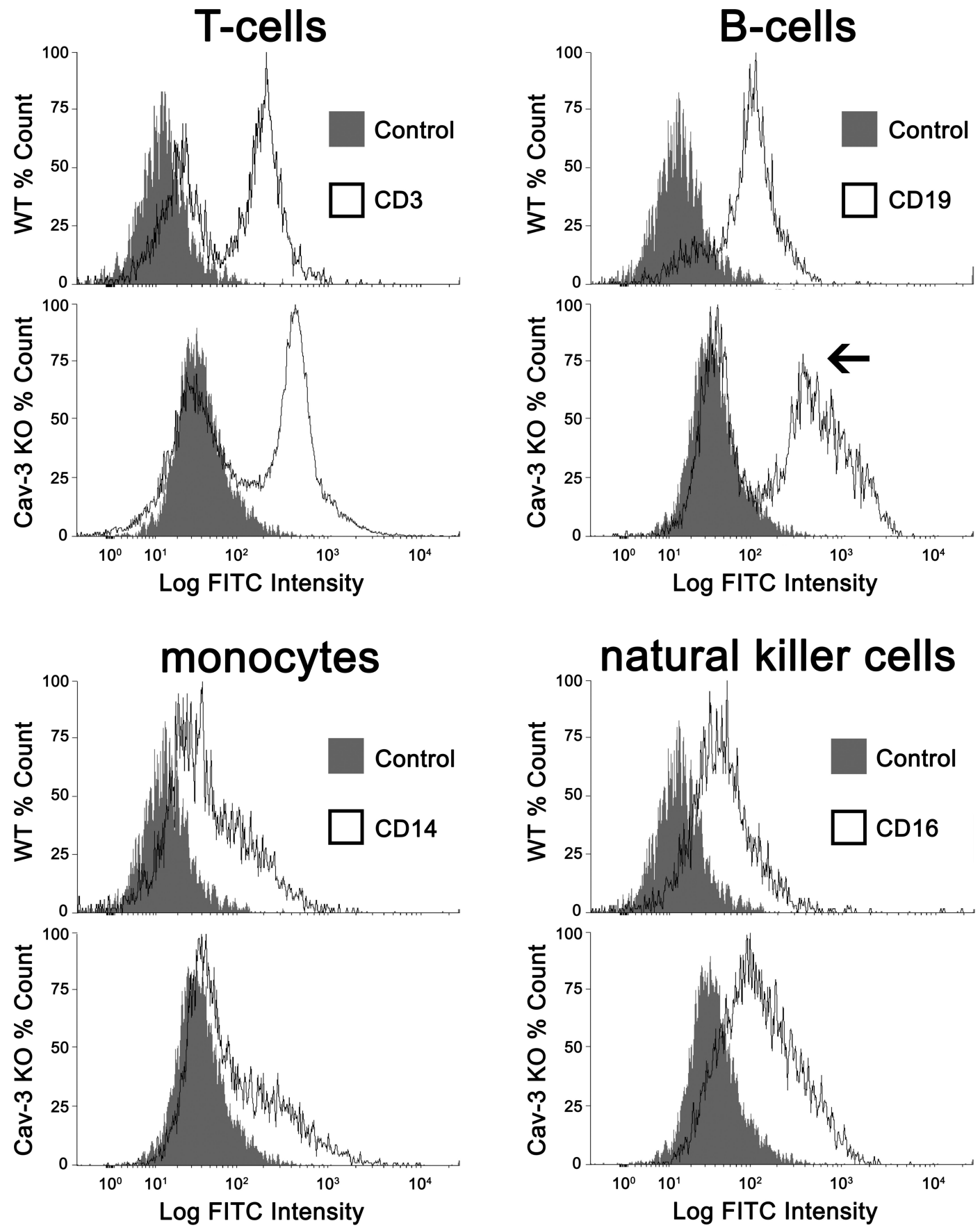

Fig. 1.

Lymphocyte/leukocyte subpopulations in Cav-3 KO mice. Monoclonal antibodies conjugated to Alexa-488 were used to identify surface antigens specific to different lymphocyte/leuckocyte populations from spleens of WT and Cav-3 KO mice by flow cytometry. B-cell population-percentage was observed to be decreased in Cav-3 KO mice (indicated by arrow) relative to WT mice. The population-percentage of monocytes, natural killer cells and T-cells remained unchanged. 

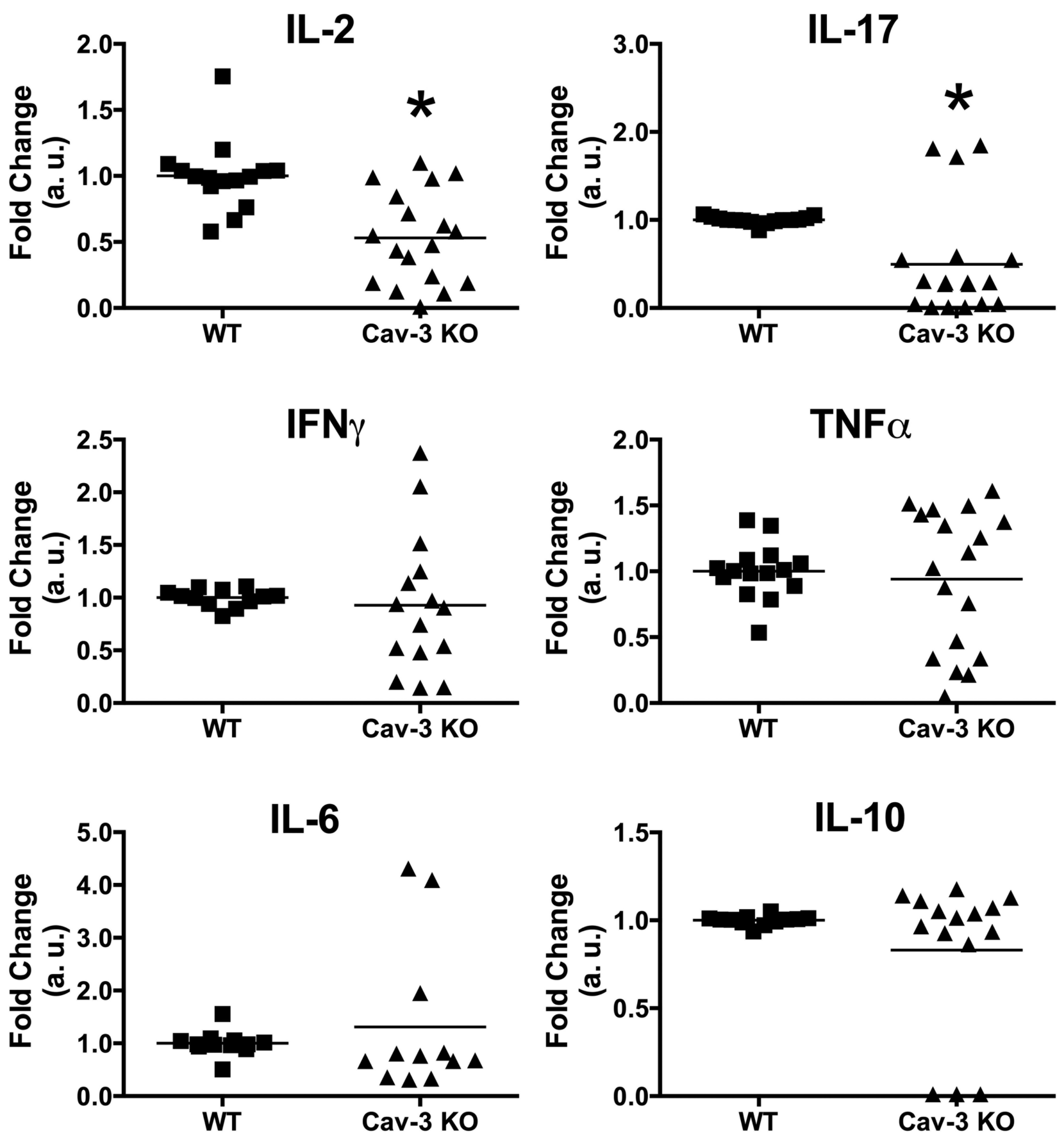

Fig. 2.

$\mathrm{T}$-cell activation and cytokine production is altered in Cav-3 $\mathrm{KO}$ mice. Lymphocytes isolated from Cav-3 KO and WT mice were stimulated by anti-CD28 and CD3 antibodies to induce activation of T-cells. Lymphocytes from Cav-3 KO mice exhibited a decrease in IL-2 and IL-17 production relative to lymphocytes from WT mice. $*=p<0.05$ from control. 